\title{
Endovascular Management of Unintentional Thoracic Aorta Injury
}

\author{
A Arrichiello MD' ${ }^{1}$, AM lerardi MD², SA Angileri MD², L Di Meglio MD \\ and G Carrafiello MD²,3
}

\author{
'Postgraduation School in Radiodiagnostics, Università degli Studi di Milano, Via Festa del Perdono 7, 20122, Milan, Italy \\ 2Operative Unit of Radiology, Fondazione IRCCS Ca' Granda Ospedale Maggiore Policlinico di Milano, Via Francesco Sforza 35 , \\ 20122, Milano, Italy \\ ${ }^{3}$ Department of Health Sciences, Università degli Studi di Milano, Via Festa del Perdono 7, 20122, Milan, Italy
}

Keywords: TEVAR; Emergency; Endovascular; Thoracic Aorta; Penetrating Trauma; latrogenic; Complication; Ablation; Bleeding

We report on a case of a major complication following a microwave (MW) ablation of a hepatic lesion, which was successfully managed using an endovascular approach.

Bleeding is one of the most common major complications of hepatic tumor ablation, although direct injury of the thoracic aorta is extremely rare [1]. Immediately after the procedure, the patient presented with pain, tachycardia and hypotension. Ultrasonic intraprocedural control of the Morison's pouch was negative, but the patient didn't recover after medical therapy. Thus, an angio-computed tomography (CT) was performed.

The coronal maximum intensity projection (MIP) images of the CT were very impressive, showing a voluminous blush from the thoracic aorta feeding a massive hematoma (Figure 1). Wasting no time is crucial in these cases, and as soon as the complex injury was appreciated, the patient was immediately transferred to the Angio-Suite. An endovascular approach is a well-established treatment for thoracic aortic lesions [2].

Interventional radiologists and vascular surgeons worked together to solve this complex case. After bilateral femoral access, an angiography of the aorta was

\section{Corresponding author:}

Antonio Arrichiello, Postgraduate School in Radiodiagnostics, Università degli Studi di Milano, Via Festa del Perdono 7, 20122, Milan, Italy.

Email: arrichielloantonio@gmail.com

(C) 2021 CC BY 4.0 - in cooperation with Depts. of Cardiothoracic/ Vascular Surgery, General Surgery and Anesthesia, Örebro University Hospital and Örebro University, Sweden

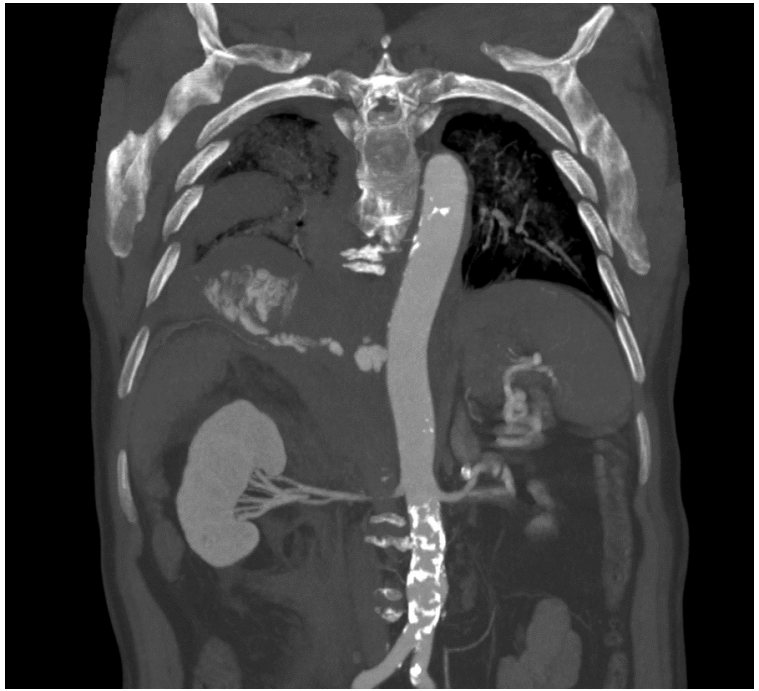

Figure 1 Coronal MIP image of the thoracic aorta showing a massive blush and hemothorax.

performed using a pig-tail catheter. The angiography showed the active blush from the aorta (Figure 2). A "Gore® TAG®" stent graft was immediately placed above the celiac trunk, excluding the thoracic injury. The final angiography showed a perfect placement of the stent graft, with no more vascular blush (Figure 3).

The patient was dismissed after 2 weeks, in good condition. A follow-up CT after a month showed perfect placement of the prothesis and quite total resolution of the hematoma (Figure 4). In a life-risk situation, nerve, haste and a multidisciplinary approach are crucial to save the patient's life. 


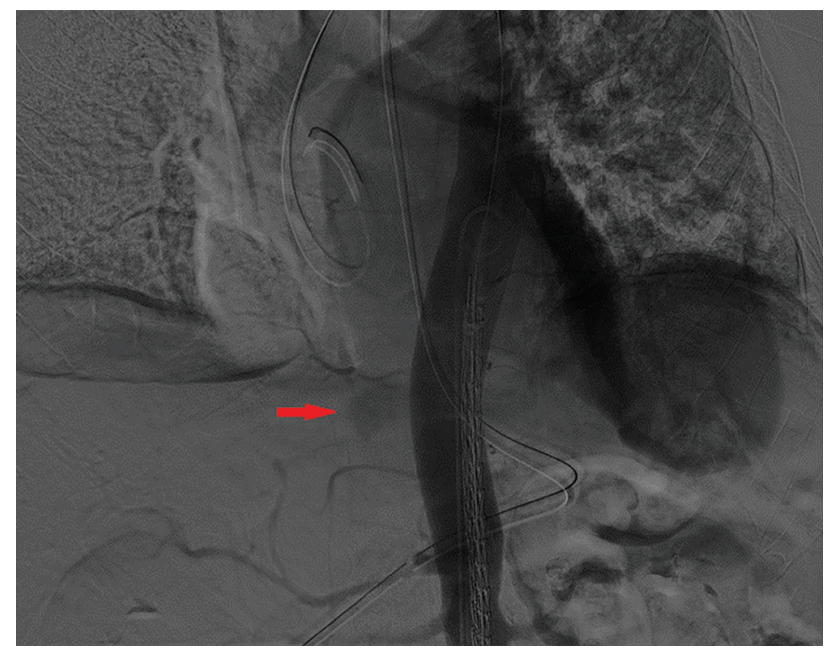

Figure 2 Aortography showing the massive blush (red arrow).

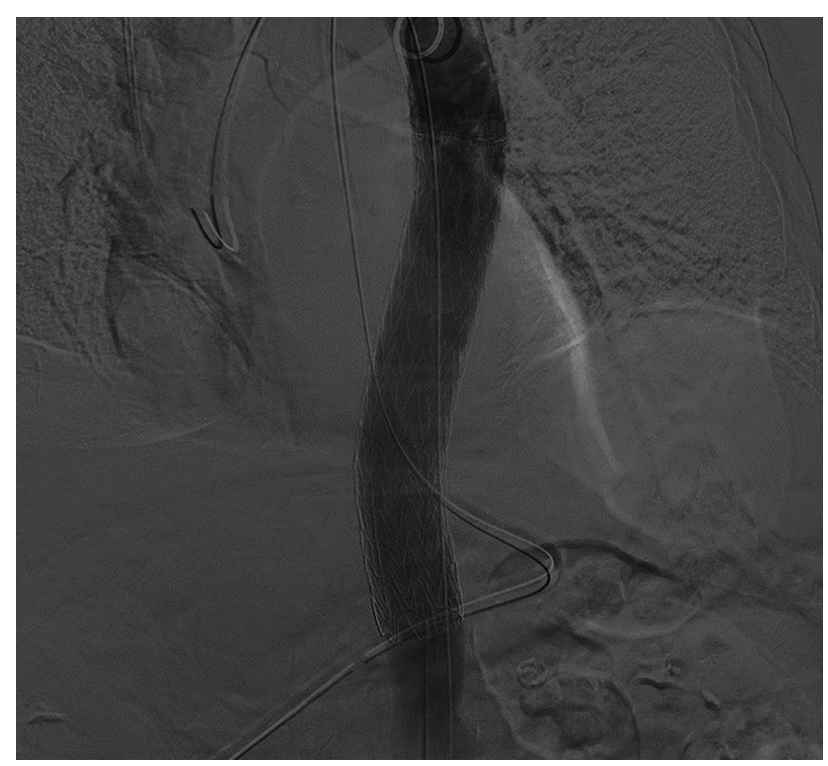

Figure 3 Post-procedure aortography, confirming the right placement of the stent graft.

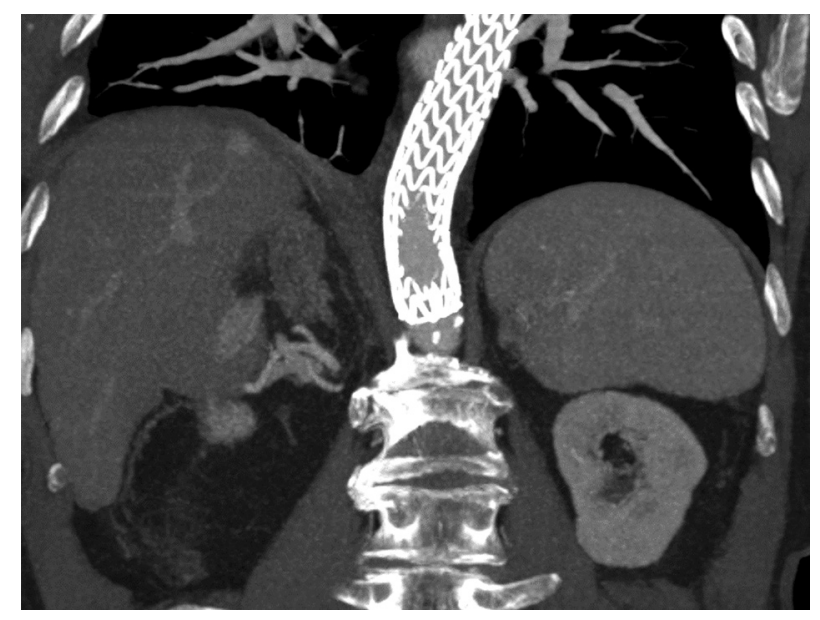

Figure 4 Coronal MIP image of the follow-up angio-computed tomography after 1 month.

\section{Ethical Approval and Informed Consent}

All procedures performed in studies involving human participants were in accordance with the ethical standards of the institutional and/or national research committee and with the 1964 Helsinki declaration and its later amendments or comparable ethical standards. Written informed consent to the CT and procedure was obtained from all subjects in this study.

\section{Ethics Statement}

(1) All the authors mentioned in the manuscript have agreed to authorship, read and approved the manuscript, and given consent for submission and subsequent publication of the manuscript.

(2) The authors declare that they have read and abided by the JEVTM statement of ethical standards including rules of informed consent and ethical committee approval as stated in the article.

\section{Conflicts of Interest}

The authors declare that they have no conflicts of interest and have no financial relationship with any sponsoring organization.

\section{Funding}

The authors received no financial support for the research, authorship, and/or publication of this article.

\section{Author Contributions}

All the authors contributed to the selection of the images and the production of the manuscript.

\section{REFERENCES}

[1] Lahat E, Eshkenazy R, Zendel A, et al. Complications after percutaneous ablation of liver tumors: a systematic review. Hepatobiliary Surg Nutr. 2014;3(5):317-323.

[2] Piffaretti G, Carrafiello G, Ierardi AM, et al. Thoracic endovascular aortic repair for blunt thoracic aortic injuries in complex aortic arch vessels anatomies. Ann Vasc Surg. 2015;29(6):1320.e11-1320.e1.32E15. 